\title{
Spontaneously axisymmetry breaking phase in a binary mixture of spinor Bose-Einstein condensates
}

\author{
Z. F. Xu, ${ }^{1}$ J. W. Mei, ${ }^{2}$ R. Lü, ${ }^{1}$ and L. You ${ }^{1}$ \\ ${ }^{1}$ Department of Physics, Tsinghua University, Beijing 100084, People's Republic of China \\ ${ }^{2}$ Institute for Advanced Study, Tsinghua University, Beijing 100084, People's Republic of China
}

(Dated: November 11, 2018)

\begin{abstract}
We study the ground state phases for a mixture of two atomic spin-1 Bose-Einstein condensates (BECs) in the presence of a weak magnetic (B-) field. The ground state is found to contain a broken-axisymmetry (BA) phase due to competitions among intra- and inter-species spin exchange interactions and the linear Zeeman shifts. This is in contrast to the case of a single species spin1 condensate, where the axisymmetry breaking results from competitions among the linear and quadratic Zeeman shifts and the intra-species ferromagnetic interaction. All other remaining ground state phases for the mixture are found to preserve axisymmetry. We further elaborate on the ground state phase diagram and calculate their Bogoliubov excitation spectra. For the BA phase, there exist three Goldstone modes attempting to restore the broken $\mathrm{U}(1)$ and $\mathrm{SO}(2)$ symmetries.
\end{abstract}

PACS numbers: 03.75.Mn, 03.75.Kk, 67.60.Bc

\section{INTRODUCTION}

The spin degrees of freedom is described by the $\mathrm{SU}(2)$ symmetry group, which can take different irreducible representations, each corresponding to different values of the spin. For an atom in a hyperfine spin $f$ state, its corresponding $\mathrm{SU}(2)$ representation is $2 f+1$ dimension. With the help of optical traps, spinor condensates with atoms in all these representations have been realized in many different atomic species and states $1[6]$.

Extensive experimental and theoretical investigations have focused mainly on atomic spinor condensates with spin equal to 1,2 , and 3 . For the simplest case of spin-1 condensates 7 -11], the mean-field ground states [7, 12], and exact full quantum eigenstates [12, 13] are intensively explored, also noted is the possibility of topological fractionalized 1/2-vortex excitations in the antiferromagnetic phase [14] and the appearance and formation of spin domains in the ferromagnetic phase [15, 16. In addition, theoretical studies [9, 17, 18] either based on mean-field approximation or including quantum spin fluctuations, and experimental efforts [19, 20] have been made to investigate the spin dynamics of the condensate. Similar efforts are directed at spin-2 condensates [12, 21, 22], whose ground states contain one extra phase: the cyclic phase, with an exotic symmetry described by the non-Abelian tetrahedral group $\mathrm{T}$, potentially of significant value for demonstrating various topological excitations $23-25]$. Under the single spatial mode approximation (SMA), the exact eigenspectra and eigenstates of a spin-2 condensate are derived [12, 22], which shows that the ground state is in general fragmented. The spin dynamics are also studied in the presence of a B-field, with the linear and quadratic Zeeman shifts 5, 26, 27]. For the case of the spin-2 hyperfine manifold of a ${ }^{87} \mathrm{Rb}$ condensate 27], its spin dynamics show amplitude resonance when the B-field is tuned experimentally just like spin mixing in a spin-1 condensate [17, 18]. When the spin increases to 3 [6], the ground state contains many more phases [28, 29], and new possibilities for richer and more colorful physics become available, waiting to be further explored.

Parallel to these studies of spinor condensates, the efforts are multi-component quantum gases have also received equal attention, most notably the case of pseudo spin-1/2 condensates [30 35] involving two atomic species or components. Since the total spin is conserved under the density dependent interaction between the two components, even in the presence of evaporative cooling if the interaction is $\mathrm{SU}(2)$ symmetric, A. B. Kuklov et al. [33] predicted that in the ground states all atoms condense into two orthogonal spatial orbitals, resulting in a maximally entangled many atom state. Replacing the two orbitals with two different species of atoms, Shi et al. 35] found a ground state condensate with an entangled order parameter. For two bosonic species of spin1 atoms, such as ${ }^{87} \mathrm{Rb}$ and ${ }^{23} \mathrm{Na}$ atoms in the lowest hyperfine state manifold, the mixture reduces to a binary mixture of spin-1 BECs. In the absence of external B-field, the ground state phases are essentially known, from the semi-classical phase diagram under the meanfield approximation [36] to the quantum results under the SMA for each of the two species and including quantum spin fluctuations [37 39]. Several interesting results have been revealed already. For example, the ground state reduces to a maximally entangled states [37, 39] where the mixture becomes fragmented and the number fluctuations [38] exhibit drastically different features from the isolated spin- 1 condensates, when the inter-species' antiferromagnetic spin exchange interaction is large enough and when the inter-species singlet pairing interaction is ignored.

In this work, we continue our study of a binary mixture of spin-1 condensates, in the presence of a weak external B-field. First, as in all experimental setups, a nonzero B-field inevitably exists no matter how small it may be. Second, it is motivated by the possibilities of interesting 
physics due to the competitions between the linear and quadratic Zeeman shifts and the ferromagnetic interactions, which for the case of isolated spin-1 condensate is shown to induce a spontaneous axisymmetry breaking phase [3, 40]. We find that by tuning the inter-species spin exchange interaction and the linear Zeeman shift, and largely ignoring the quadratic Zeeman shifts (because the B-field is weak), there exists an analogous BA phase in a system of binary mixture of spin-1 condensates.

This article is organized as follows. In section II, we introduce the model formulation for a binary mixture of spin- 1 condensates in the presence of an external Bfield. Sec. III is devoted to the mean field results of the broken-axisymmetry (BA) phase, where we discuss the special case and the general case respectively. In Sec. IV, we elaborate on the Bogoliubov spectra for the various quantum phases of the mixture, and we point out the interesting zero energy Goldstone modes responsible for restoring the broken continuous symmetries: $\mathrm{U}(1)$ and $\mathrm{SO}(2)$. Also we propose a new scheme for classifying the various phases in the ground states using the number of nonzero components for their associated order parameters. Finally we conclude in Sec. V. The appendix provides some mathematical details.

\section{OUR MODEL}

In this section, we first review the basic formulation introduced earlier for the inter-species atomic interactions. Between two distinguishable spin-1 atoms (of different species), they can be parameterized by the three different scattering lengths $a_{0,1,2}^{(12)}$ which refer to three channels of total spin $F_{\text {tot }}=0,1$, and 2 respectively. Define $g_{0,1,2}^{(12)}=4 \pi \hbar^{2} a_{0,1,2}^{(12)} / \mu$ with $\mu=M_{1} M_{2} /\left(M_{1}+M_{2}\right)$ the reduced mass and $M_{l}$ the mass for each atomic species $(l=1,2)$, the corresponding pseudo-potential is given by $V_{12}\left(\mathbf{r}_{1}-\mathbf{r}_{2}\right)=\left(g_{0}^{(12)} \mathcal{P}_{0}+g_{1}^{(12)} \mathcal{P}_{1}+g_{2}^{(12)} \mathcal{P}_{2}\right) \delta\left(\mathbf{r}_{1}-\mathbf{r}_{2}\right) / 2$. $\mathcal{P}_{0,1,2}$ is the corresponding total spin projection operator, and $\vec{F}_{1} \cdot \vec{F}_{2}=\mathcal{P}_{2}-\mathcal{P}_{1}-2 \mathcal{P}_{0}$. The inter-species interaction is then expressed as

$$
V_{12}\left(\mathbf{r}_{1}-\mathbf{r}_{2}\right)=\frac{1}{2}\left(\alpha+\beta \mathbf{F}_{1} \cdot \mathbf{F}_{2}+\gamma \mathcal{P}_{0}\right) \delta\left(\mathbf{r}_{1}-\mathbf{r}_{2}\right),
$$

where $\alpha=\left(g_{1}^{(12)}+g_{2}^{(12)}\right) / 2, \beta=\left(-g_{1}^{(12)}+g_{2}^{(12)}\right) / 2$, and $\gamma=\left(2 g_{0}^{(12)}-3 g_{1}^{(12)}+g_{2}^{(12)}\right) / 2$. Denoting $\hat{\Psi}_{M_{F}}(\mathbf{r})$ and $\hat{\Phi}_{M_{F}}(\mathbf{r})$ as the two species annihilation field operator at a position $\mathbf{r}$ respectively, the Hamiltonian of a homogenous system of binary mixture under B-field becomes

$$
\begin{aligned}
\hat{H}= & \hat{H}_{1}+\hat{H}_{2}+\hat{H}_{12}, \\
\hat{H}_{1}= & \int d \mathbf{r}\left\{\hat{\Psi}_{m}^{\dagger}\left(-\frac{\hbar^{2}}{2 M_{1}} \nabla^{2}-p_{1} m+q_{1} m^{2}\right) \hat{\Psi}_{m}\right. \\
& \left.+\frac{\alpha_{1}}{2} \hat{\Psi}_{i}^{\dagger} \hat{\Psi}_{j}^{\dagger} \hat{\Psi}_{j} \hat{\Psi}_{i}+\frac{\beta_{1}}{2} \hat{\Psi}_{i}^{\dagger} \hat{\Psi}_{k}^{\dagger} \mathbf{F}_{1 i j} \cdot \mathbf{F}_{1 k l} \hat{\Psi}_{l} \hat{\Psi}_{j}\right\} \\
\hat{H}_{12}= & \frac{1}{2} \int d \mathbf{r}\left\{\alpha \hat{\Psi}_{i}^{\dagger} \hat{\Phi}_{j}^{\dagger} \hat{\Phi}_{j} \hat{\Psi}_{i}\right. \\
& \left.+\beta \hat{\Psi}_{i}^{\dagger} \hat{\Phi}_{k}^{\dagger} \mathbf{F}_{1 i j} \cdot \mathbf{F}_{2 k l} \hat{\Phi}_{l} \hat{\Psi}_{j}+\frac{1}{3} \gamma \hat{s}^{\dagger} \hat{s}\right\},
\end{aligned}
$$

where $p_{1}$ and $q_{1}$ are the linear and the quadratic Zeeman shifts, respectively, and summation over repeated indices are assumed. $\hat{H}_{2}$ has the same expression as $\hat{H}_{1}$ except for the substitution of subscript 1 by 2 and $\hat{\Psi}$ by $\hat{\Phi} . \quad \hat{s}=\left(\hat{\Psi}_{1} \hat{\Phi}_{-1}-\hat{\Psi}_{0} \hat{\Phi}_{0}+\hat{\Psi}_{-1} \hat{\Phi}_{1}\right)$ is the inter-species singlet pairing operator.

\section{MEAN-FIELD GROUND STATES}

This study concerns the limit of weak external B-fields, when the quadratic Zeeman shifts can be neglected, or $q_{1}=q_{2}=0$. Without loss of generality we assume $p_{1} p_{2} \geq 0$. For the mixture of two alkali species, such as the spin- $1{ }^{87} \mathrm{Rb}$ and ${ }^{23} \mathrm{Na}$ condensates, under the assumption of interspecies interaction mainly arise from the contributions of the two valence electrons [41 43], we can approximate $\gamma=0$ [44]. For a uniform system, it is convenient to expand the field operators in terms of plane waves as $\hat{\Psi}_{m}=\Omega^{-1 / 2} \sum_{\mathbf{k}} e^{i \mathbf{k} \cdot \mathbf{r}} \hat{a}_{\mathbf{k}, m}$ and $\hat{\Phi}_{m}=\Omega^{-1 / 2} \sum_{\mathbf{k}} e^{i \mathbf{k} \cdot \mathbf{r}} \hat{b}_{\mathbf{k}, m}$, where $\Omega$ is the system volume, and $\hat{a}_{\mathbf{k}, m}\left(\hat{b}_{\mathbf{k}, m}\right)$ denotes the annihilation operator of an atom of species one (two) with momentum $\hbar \mathbf{k}$ and magnetic quantum number $m$. Then the Hamiltonian of Eq. (2) can be rewritten as

$$
\begin{aligned}
\hat{H}_{1}= & \sum_{\mathbf{k}, m}\left(\varepsilon_{1 \mathbf{k}}-p_{1} m\right) \hat{a}_{\mathbf{k}, m}^{\dagger} \hat{a}_{\mathbf{k}, m}+\frac{\alpha_{1}}{2 \Omega} \sum_{\mathbf{k}}: \hat{\rho}_{1, \mathbf{k}} \hat{\rho}_{1,-\mathbf{k}}: \\
& +\frac{\beta_{1}}{2 \Omega} \sum_{\mathbf{k}}: \hat{\mathbf{f}}_{1, \mathbf{k}} \cdot \hat{\mathbf{f}}_{1,-\mathbf{k}}: \\
\hat{H}_{12}= & \frac{\alpha}{2 \Omega} \sum_{\mathbf{k}} \hat{\rho}_{1, \mathbf{k}} \hat{\rho}_{2,-\mathbf{k}}+\frac{\beta}{2 \Omega} \sum_{\mathbf{k}} \hat{\mathbf{f}}_{1, \mathbf{k}} \cdot \hat{\mathbf{f}}_{2,-\mathbf{k}}
\end{aligned}
$$

where $\varepsilon_{1 \mathbf{k}}=\hbar^{2} \mathbf{k}^{2} / 2 M_{1}, \hat{\rho}_{1, \mathbf{k}}=\sum_{\mathbf{q}, m} \hat{a}_{\mathbf{q}+\mathbf{k}, m}^{\dagger} \hat{a}_{\mathbf{q}, m}$, and $\hat{\mathbf{f}}_{1, \mathbf{k}}=\sum_{\mathbf{q}, m, n} \hat{a}_{\mathbf{q}+\mathbf{k}, m}^{\dagger} \mathbf{F}_{1 m n} \hat{a}_{\mathbf{q}, n}$. The symbol : : represents the normal ordering of the operators. Similar to Eq. (2), $\hat{H}_{2}$ is given by replacing the subscript 1 by 2 , and the operator $\hat{a}$ by $\hat{b}$ in $\hat{H}_{1}$.

The condensate component corresponds to the zero momentum $\mathbf{k}=0$ state, which is occupied by a macroscopic number of atoms. The corresponding Hamiltonian 
becomes

$$
\begin{aligned}
\hat{H}_{\mathrm{BEC}} & =\sum_{m}-m\left(p_{1} \hat{a}_{\mathbf{0}, m}^{\dagger} \hat{a}_{\mathbf{0}, m}+p_{2} \hat{b}_{\mathbf{0}, m}^{\dagger} \hat{b}_{\mathbf{0}, m}\right) \\
& +\frac{\alpha_{1}}{2 \Omega}: \hat{\rho}_{1, \mathbf{0}}^{2}:+\frac{\beta_{1}}{2 \Omega}: \hat{\mathbf{f}}_{1, \mathbf{0}}^{2}:+\frac{\alpha_{2}}{2 \Omega}: \hat{\rho}_{2, \mathbf{0}}^{2}: \\
& +\frac{\beta_{2}}{2 \Omega}: \hat{\mathbf{f}}_{2, \mathbf{0}}^{2}:+\frac{\alpha}{2 \Omega} \hat{\rho}_{1, \mathbf{0}} \hat{\rho}_{2, \mathbf{0}}+\frac{\beta}{2 \Omega} \hat{\mathbf{f}}_{1, \mathbf{0}} \cdot \hat{\mathbf{f}}_{2, \mathbf{0}}
\end{aligned}
$$

Under the mean-field approximation, the operator $\hat{a}_{\mathbf{0}, m}$ $\left(\hat{b}_{\mathbf{0}, m}\right)$ is replaced by $c$-number $\sqrt{N}_{1} \zeta_{1, m}\left(\sqrt{N}_{2} \zeta_{2, m}\right)$, where $N_{1}, N_{2}$ are the atom numbers for the species one and two respectively, and $\zeta_{1}=\left(\zeta_{1,1}, \zeta_{1,0}, \zeta_{1,-1}\right)^{T}$ and $\zeta_{2}=\left(\zeta_{2,1}, \zeta_{2,0}, \zeta_{2,-1}\right)^{T}$ are normalized spin-1 spinors. As a result, in the mean-field approximation the ground state spinor wavefunction is found by minimizing the mean-field spin-dependent energy given by

$$
\begin{aligned}
\mathcal{E}_{s}= & -p_{1}\left\langle F_{1 \mathrm{z}}\right\rangle+\frac{1}{2} \beta_{1} n_{1}\left\langle\mathbf{F}_{1}\right\rangle^{2}-p_{2} \frac{N_{2}}{N_{1}}\left\langle F_{2 \mathrm{z}}\right\rangle \\
& +\frac{1}{2} \beta_{2} n_{2} \frac{N_{2}}{N_{1}}\left\langle\mathbf{F}_{2}\right\rangle^{2}+\frac{1}{2} \beta n_{2}\left\langle\mathbf{F}_{1}\right\rangle \cdot\left\langle\mathbf{F}_{2}\right\rangle \\
= & -p f_{1 \mathrm{z}}-x p f_{2 \mathrm{z}}+\frac{1}{2}\left(\beta_{1}^{\prime} \mathbf{f}_{1}^{2}+\beta_{2}^{\prime} \mathbf{f}_{2}^{2}+\beta^{\prime} \mathbf{f}_{1} \cdot \mathbf{f}_{2}\right),
\end{aligned}
$$

where $n_{j}=N_{j} / \Omega$ is the condensate density of the $j$ th species and $\mathbf{f}_{1,2}=\left\langle\mathbf{F}_{1,2}\right\rangle, p_{1}=p_{2} N_{2} /\left(N_{1} x\right)=p$, $\beta_{1}^{\prime}=\beta_{1} n_{1}, \beta_{2}^{\prime}=\beta_{2} n_{2} N_{2} / N_{1}$ and $\beta^{\prime}=\beta n_{2}$. The procedure to find the mean-field ground states is summarized in the appendix $\mathrm{A}$, where we discuss the structure of the ground states classified into two classes: with or without broken-axisymmetry. According to Ref. [1], a state with a nonzero transverse magnetization is called a broken-axisymmetry phase. Analogously states preserving axisymmetry refer to those with zero transverse magnetization. In the BA phase, both species are found to be fully polarized. For axisymmetry preserved ground states, the wave functions take the form

$$
\zeta_{j}=e^{i \chi_{j}}\left(\begin{array}{c}
e^{i \varphi_{j}} \sqrt{\left(1+f_{\mathrm{jz}}\right) / 2} \\
0 \\
\sqrt{\left(1-f_{\mathrm{jz}}\right) / 2}
\end{array}\right),
$$

where $\chi_{j}$ and $\varphi_{j}$ are arbitrary phase angles, and $j=1,2$. When $f_{1 \mathrm{z}}=0$ or $f_{2 \mathrm{z}}=0$, the ground states include an infinite family of degenerate states with that in Eq. (6). More rigorously, we note that the nematic order for the above state (6) actually is not axisymmetric [1]. For the $\mathrm{BA}$ phase, the ground state wave functions take the form

$$
\zeta_{j}=e^{i \chi_{j}}\left(\begin{array}{c}
e^{-i \varphi_{j}} \cos ^{2} \frac{\theta_{j}}{2} \\
\sqrt{2} \cos \frac{\theta_{j}}{2} \sin \frac{\theta_{j}}{2} \\
e^{i \varphi_{j}} \sin ^{2} \frac{\theta_{j}}{2}
\end{array}\right),
$$

where $\chi_{j}$ and $\varphi_{j}$ are arbitrary phase angles, and $\varphi_{2}=$ $\varphi_{1}+\pi(\bmod 2 \pi), j=1,2 . \theta_{1}$ and $\theta_{2}$ are determined by $f_{1 z}$ and $f_{2 \mathrm{z}}$ respectively as $\cos \theta_{1}=f_{1 \mathrm{z}}$ and $\cos \theta_{2}=f_{2 \mathrm{z}}$. The ground state phase diagram is characterized into three cases according to the intra-species spin exchange interaction parameter $\beta_{1}^{\prime}$ and $\beta_{2}^{\prime}$. Without loss of generality, in the following we assume $\left|\beta_{1}^{\prime}\right|<\left|\beta_{2}^{\prime}\right|$.

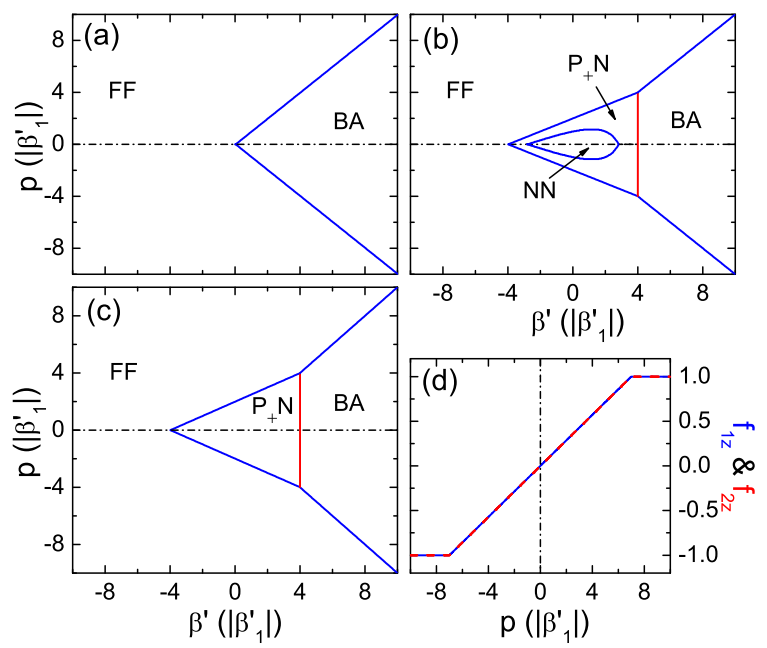

FIG. 1: (Color online). The ground state phase diagram of our model system of a binary spin- 1 condensate mixture at fixed values of $\beta_{1}^{\prime}$ and $\beta_{2}^{\prime}$ for the special case of $x=1$. The black dash-dotted lines correspond to $p=0$, which serve as guides for the eye. Blue (Red) lines denote continuous (discontinuous) phase boundaries. The first three subplots denote fixed intra-specie spin exchange interaction parameters at $\left(\beta_{1}^{\prime}\right.$, $\left.\beta_{2}^{\prime}\right) /\left|\beta_{1}^{\prime}\right|=$ : (a) $(-1,-2)$; (b) $(1,2)$; and (c) $(-1,2)$. The last one (d) illustrates the dependence of ground state order parameters $f_{1 \mathrm{z}}$ (blue solid line) and $f_{2 \mathrm{z}}$ (red dashed line) of the BA phase on the linear Zeeman shift $p$ at a fixed value of $\beta^{\prime}=7\left|\beta_{1}^{\prime}\right|$.

\section{A. the special case of $x=1$}

In this subsection, we consider the special case of $x=$ 1 , where the parameter defined as the partical number multiplying the linear Zeeman shift, of the two species are equal $\left(N_{1} p_{1}=N_{2} p_{2}\right)$. The ground states are as shown in the Fig. (1), where the first three subplots denote fixed intra-specie spin exchange interaction parameters at $\left(\beta_{1}^{\prime}\right.$, $\left.\beta_{2}^{\prime}\right) /\left|\beta_{1}^{\prime}\right|=$ : (a) $(-1,-2)$; (b) $(1,2)$; and (c) $(-1,2)$. The last one (d) illustrates the dependence of ground state order parameters $f_{1 \mathrm{z}}$ and $f_{2 \mathrm{z}}$ of the $\mathrm{BA}$ phase on the linear Zeeman shift $p$ at a fixed value of $\beta^{\prime}=7\left|\beta_{1}^{\prime}\right|$.

First, we discuss the case when the two spin- 1 condensates are both ferromagnetic $\left(\beta_{1}^{\prime}<0\right.$ and $\left.\beta_{2}^{\prime}<0\right)$. The ground state has two phases: the FF phase and the BA phase. The FF phase is a typical axisymmetry preserving phase with $f_{1 \mathrm{z}}=f_{2 \mathrm{z}}=\operatorname{sign}(p)$, which is the same as that found in [36] with atomic spins in each species fully polarized and aligned parallel to each other with $\mathbf{f}_{1}^{2}=\mathbf{f}_{2}^{2}=\mathbf{f}_{1} \cdot \mathbf{f}_{2}=1$. In the BA phase, the two spin vectors of each species are remain fully polarized but now tilted at an angle $\theta_{1}$ and $\theta_{2}$ with $\theta_{1}=\theta_{2}=\arccos \left(p / \beta^{\prime}\right)$ with respect to the $z$-axis. This is illustrated in the Fig. 2. From Eq. (A3), we confirm that in this case the BA phase exists only when $\beta^{\prime} \geq 0$, which is consistent with numerical and analytical results shown in the Fig. 1(a). The boundary between the FF phase and the BA phase can be derived by comparing the energy of Eq. (5), which 


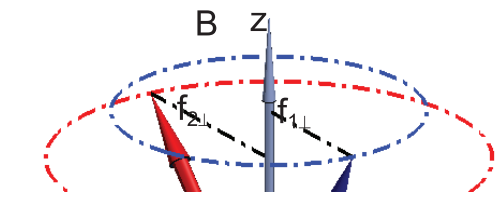

$\theta_{2} \theta_{1}$

$\mathbf{X}$

y

FIG. 2: (Color online). A schematic illustration of the spin vectors for both condensate species in the brokenaxisymmetry phase. The weak external B-field is along the $z$-axis direction. The blue (red) arrow denotes the spin vector of species one (two), which tilts at an angle with respect to the $z$-axis. The two spin vectors and the $z$-axis are in the same plane. The blue and red dotted dash lines correspond to the orbits of the end points of two spin vectors in the degenerate ground state of the broken-axisymmetry phase. The black dotted dash lines represent the transverse magnetizations $f_{1 \perp}$ and $f_{2 \perp}$ of two species respectively.

turns out to be $|p|=\beta^{\prime}$. In Fig. 1(d), we present the dependence of the order parameters $f_{1 z}$ and $f_{2 z}$ on the linear Zeeman shift $p$ at a fixed value of $\beta^{\prime}=7\left|\beta_{1}^{\prime}\right|$. In the region of $|p| \leq \beta^{\prime}, f_{1 \mathrm{z}}$ and $f_{2 \mathrm{z}}$ change smoothly from -1 to 1 simultaneously.

We then turn to another case when one of the spin-1 condensates is ferromagnetic $\left(\beta_{1}^{\prime}<0\right)$ and the other is antiferromagnetic or polar $\left(\beta_{2}^{\prime}>0\right)$. In the absence of Bfield, the competition between the intra- and inter-species spin exchange interactions gives rise to three phases 36 . When $\beta<-2 \beta_{2}$, the ground state is in the FF phase. Increasing the value of $\beta$, there arises another phase, where the spin vector in the ferromagnetic one is fully polarized, and the other in the polar one is not fully polarized, giving rise to $\mathbf{f}_{1}^{2}=1, \mathbf{f}_{2}^{2}=\left(\beta^{\prime} / 2 \beta_{2}^{\prime}\right)^{2}$, and $\mathbf{f}_{1} \cdot \mathbf{f}_{2}=-\beta / 2 \beta_{2}$. In the other limit when $\beta^{\prime}>2 \beta_{2}^{\prime}$, the ground state is in the AA phase where the two spin vectors are fully polarized into opposite directions with $\mathbf{f}_{1}^{2}=\mathbf{f}_{2}^{2}=1$ and $\mathbf{f}_{1} \cdot \mathbf{f}_{2}=-1$. In the presence of a weak external B-field, these three phases for the ground state in the phase space will extend into three regions. The first one is the FF phase, which has the same properties as that in the $p=0$ case, except now all spins are polarized along the external B-field direction. The second one corresponds to the other axisymmetry preserved phase denoted by $\mathrm{P}_{+} \mathrm{N}$, where we use symbol $\mathrm{P}$ and $\mathrm{N}$ to represent respectively the fully and non-fully polarized spin vectors of two species. The subscript + is used to clarify that the fully polarized spin is along the same direction of the B-field. The other remaining one is the BA phase, which is totally the same as that in the case of two ferromagnetic spin-1 condensates. In Fig. 11(c), we illustrate the ground state phase diagram. The boundary between the $\mathrm{FF}$ phase and the $\mathrm{BA}$ phase is the same as that in

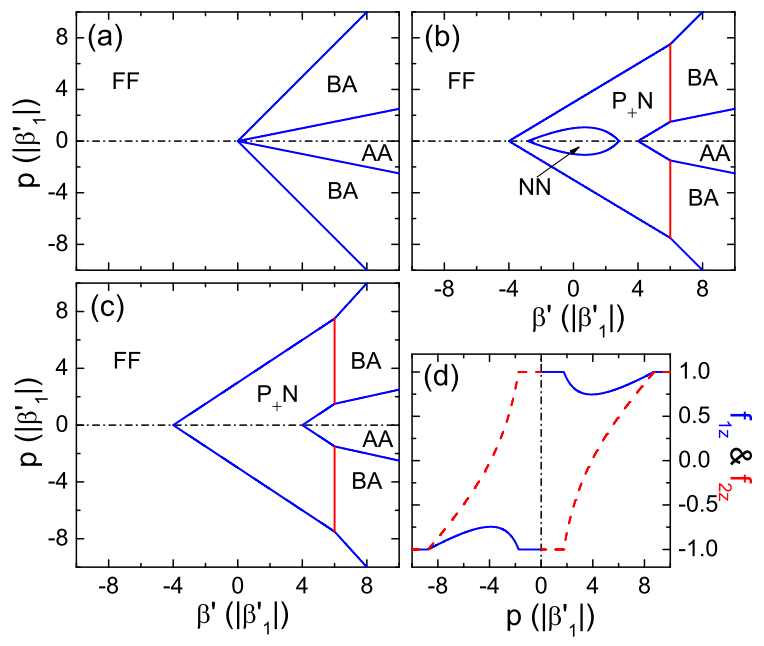

FIG. 3: (Color online). The same as in Fig. 1 but for $x=2 / 3$.

Fig. 1(a), except that when $\beta^{\prime}<2 \beta_{2}^{\prime}$, the ground state is changed to the $\mathrm{P}_{+} \mathrm{N}$ phase, which also means that the boundary between the $\mathrm{P}_{+} \mathrm{N}$ phase and the $\mathrm{BA}$ phase is $\beta^{\prime}=2 \beta_{2}^{\prime}$, which is consistent with Eq. (A3). Only when $\beta^{\prime} \geq \max \left(0,2 \beta_{1}^{\prime} x, 2 \beta_{2}^{\prime} / x\right)=2 \beta_{2}^{\prime}$ is satisfied will the BA phase possibly exist. The remaining boundary between the $\mathrm{FF}$ phase and the $\mathrm{P}_{+} \mathrm{N}$ phase satisfies the relationship $|p|=\beta_{2}^{\prime}+\beta^{\prime} / 2$. In the $\mathrm{P}_{+} \mathrm{N}$ phase, we have $f_{1 \mathrm{z}}=\operatorname{sign}(p)$ and $f_{2 \mathrm{z}}=\left(p-f_{1 \mathrm{z}} \beta^{\prime} / 2\right) / \beta_{2}^{\prime}$.

Next, we consider the last case with two spin-1 polar condensates $\left(\beta_{2}^{\prime}>\beta_{1}^{\prime}>0\right)$. In Fig. [1(b), we present the phase diagram as well as the boundaries separating the phases. Similar to the second case of Fig. 11(c), the ground state has three phases: FF, $\mathrm{P}_{+} \mathrm{N}$, and BA. The order parameters and the boundaries between these three phases are the same as that in the previous case of Fig. 11(c). The only difference arises from the competition with the intra-species interaction in the anti-ferromagnetic condensate. A new phase emerges in the center of the $\mathrm{P}_{+} \mathrm{N}$ phase, which is denoted as the NN phase. In this new phase, neither spin vectors of each species are fully polarized. The boundaries between the $\mathrm{NN}$ phase and the $\mathrm{P}_{+} \mathrm{N}$ phase satisfy the following relationships $\left(\beta^{\prime} / 2-\beta_{2}^{\prime}\right)|p|=$ $\beta^{\prime 2} / 4-\beta_{1}^{\prime} \beta_{2}^{\prime}$. For the ground states in the NN phase, we have $f_{1 \mathrm{z}}=\left(\beta^{\prime} / 2-\beta_{2}^{\prime}\right) p /\left(\beta^{\prime 2} / 4-\beta_{1}^{\prime} \beta_{2}^{\prime}\right)$ and $f_{2 \mathrm{z}}=$ $\left(\beta^{\prime} / 2-\beta_{1}^{\prime}\right) p /\left(\beta^{\prime 2} / 4-\beta_{1}^{\prime} \beta_{2}^{\prime}\right)$.

\section{B. the general case}

The ground state phase diagram for the special case of $x=1$ as discussed above contains both axisymmetry preserved and broken phases. Their boundaries can be partially determined by Eq. (A3). The AA phase is found to exist only when $p=0$. It amounts to an infinite set of degenerate states, where the two spin vectors are antiparallel while remaining fully polarized. As long as $x \neq 1$ is 


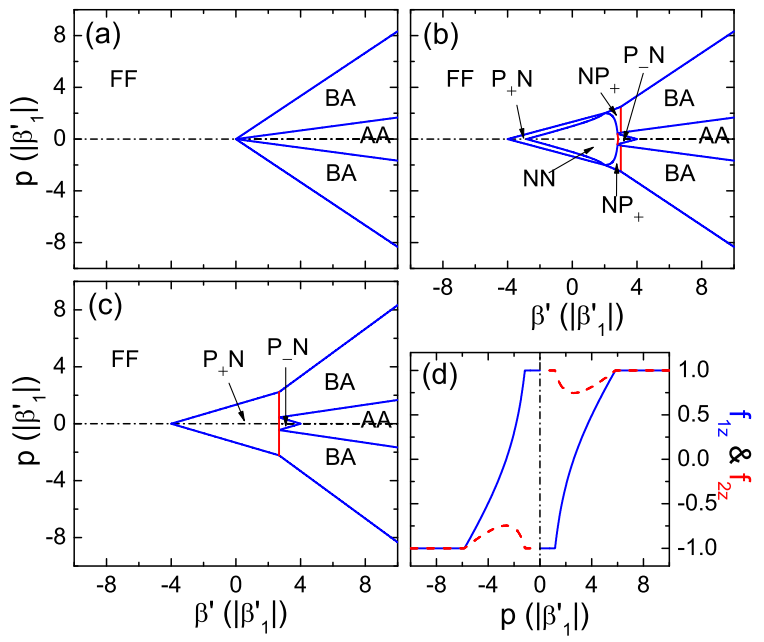

FIG. 4: (Color online). The same as in Fig. 1, but for $x=3 / 2$.

satisfied, the region of the AA phase extends to $p \neq 0$, where one spin vector will be parallel to the B-field, while the other one become antiparallel. The order parameters $\left(f_{1 \mathrm{z}}, f_{2 \mathrm{z}}\right)$ for the $\mathrm{P}_{+} \mathrm{N}, \mathrm{NN}$ and $\mathrm{BA}$ phases change into the following forms $\left(\operatorname{sign}(p),\left(2 x p-\operatorname{sign}(p) \beta^{\prime}\right) / 2 \beta_{2}^{\prime}\right)$, $\left(\left(2 x p \beta^{\prime}-4 p \beta_{2}^{\prime}\right) /\left(\beta^{\prime 2}-4 \beta_{1}^{\prime} \beta_{2}^{\prime}\right),\left(2 p \beta^{\prime}-4 x p \beta_{1}^{\prime}\right) /\left(\beta^{\prime 2}-\right.\right.$ $\left.\left.4 \beta_{1}^{\prime} \beta_{2}^{\prime}\right)\right),\left(x p / \beta^{\prime}-\left(x^{2}-1\right) \beta^{\prime} / 4 x p, p / \beta^{\prime}+\left(x^{2}-1\right) \beta^{\prime} / 4 x^{2} p\right)$, respectively.

We first consider the case with $x<1$, whose ground state phase diagram is demonstrated in Fig. 3. Due to our choice of $\left|\beta_{1}^{\prime}\right|<\left|\beta_{2}^{\prime}\right|$, the ground state phase diagram is similar to the case of $x=1$. One notable difference is that the AA phase appears in the middle of the BA phase, and the boundaries between them are given by $|p|=\beta^{\prime}(1-x) / 2 x$. And the boundary between the BA phase and FF phase is also changed to be described by $|p|=\beta^{\prime}(x+1) / 2 x$. As a result of the appearance of the AA phase, the $\mathrm{P}_{+} \mathrm{N}$ phase and the AA phase will now in touch with each other, and their boundary is given by $|p|=\left(\beta^{\prime}-2 \beta_{2}^{\prime}\right) / 2 x$. The NN phase remains immersed in the $\mathrm{P}_{+} \mathrm{N}$ phase, and their boundary satisfies $|p|=\left(\beta^{\prime 2}-4 \beta_{1}^{\prime} \beta_{2}^{\prime}\right) /\left(2 x \beta^{\prime}-4 \beta_{2}^{\prime}\right)$. In Fig. 3, we illustrate the phase diagram, where the blue (red) solid lines denote the continuous (discontinuous) phase boundaries. In Fig. 3(d), we display the order parameters of the ground states at fixed interspecies spin-exchange interaction $\beta^{\prime}=7\left|\beta_{1}^{\prime}\right|$. Due to the emergence of the AA phase in the middle of the BA phase, $f_{2 \mathrm{z}}$ changes from - 1 to 1 twice when $p$ changes from $-\infty$ to $\infty$, while $f_{1 z}$ oscillates near the maximum and minimum values respectively, which is different from the special case of $x$ discussed previously, where $f_{1 \mathrm{z}}$ and $f_{2 \mathrm{z}}$ change simultaneously from -1 to 1 .

Next we discuss the other case of $x>1$, whose ground state phase diagram is demonstrated in Fig. 4 Due to the choice of $\left|\beta_{1}^{\prime}\right|<\left|\beta_{2}^{\prime}\right|$ and $x>1$, the ground state phase diagram is much more complicated than that of $x \leq 1$. For two ferromagnetic condensates $\left(\beta_{1}^{\prime}<0\right.$ and $\beta_{2}^{\prime}<0$ ), the ground state phase diagram is similar to that of $x<1$. With one ferromagnetic $\left(\beta_{1}^{\prime}<0\right)$ and one anti-ferromagnetic condensate $\left(\beta_{2}^{\prime}>0\right)$, the phase diagram becomes different from the case of $x<1$. A new phase denoted by $\mathrm{P}_{-} \mathrm{N}$ emerges, where the subscript is used to denote a spin vector being fully anti-aligned with the external B-field. This phase arises from increased interspecies spin-exchange interaction $\beta^{\prime}$ in this case of $x>1$. Starting from the $\mathrm{P}_{+} \mathrm{N}$ phase, with the increase of $\beta^{\prime}$, the spin vector of the second species becomes incresingly polarized antiparallel to the B-field, which weights in more linear Zeeman energy contribution from the second species, and the spins for both species will flip to the opposite directions. The order parameters for the $\mathrm{P}_{-} \mathrm{N}$ phase take the forms $f_{1 \mathrm{z}}=-\operatorname{sign}(p)$ and $f_{2 \mathrm{z}}=\left(2 x p+\operatorname{sign}(p) \beta^{\prime}\right) / 2 \beta_{2}^{\prime}$. Incidently, we find that the phase boundaries between the $\mathrm{P}_{+} \mathrm{N}$ phase and the $\mathrm{BA}$ or the $\mathrm{P}_{-} \mathrm{N}$ phases are the same: described by $\beta^{\prime}=2 \beta_{2}^{\prime} / x$. The remaining phase boundaries take the same forms as that in the case of $x<1$, except for the one between the phase of $\mathrm{P}_{-} \mathrm{N}$ and $\mathrm{AA}$, which takes the form $|p|=$ $\left(2 \beta_{2}^{\prime}-\beta^{\prime}\right) / 2 x$. For the choice of two anti-ferromagnetic condensates $\left(\beta_{1}^{\prime}>0\right.$ and $\left.\beta_{2}^{\prime}>0\right)$, in addition to the $\mathrm{P}_{-} \mathrm{N}$ phase, a new phase denoted by $\mathrm{NP}_{+}$emerges between the BA phase and the NN phase. The order parameters for the $\mathrm{NP}_{+}$phase are given by $f_{1 \mathrm{z}}=\left(2 p-\operatorname{sign}(p) \beta^{\prime}\right) / 2 \beta_{1}^{\prime}$ and $f_{2 \mathrm{z}}=\operatorname{sign}(p)$. The boundaries between the $\mathrm{NP}_{+}$ phase and the NN, the AA, and the FF phases take the forma $|p|=\left(\beta^{\prime 2}-4 \beta_{1}^{\prime} \beta_{2}^{\prime}\right) /\left(2 \beta^{\prime}-4 x \beta_{1}^{\prime}\right),|p|=\beta^{\prime} / 2-\beta_{1}^{\prime}$, and $|p|=\beta^{\prime} / 2+\beta_{1}^{\prime}$, respectively. The complete phase diagram is illustrated in the Fig. 4. Additionally we illustrate the order parameters for the ground state at fixed value of $\beta^{\prime}=7\left|\beta_{1}^{\prime}\right|$. When $x>1, f_{1 \mathrm{z}}$ is observed to change from -1 to 1 twice as $p$ changes from $-\infty$ to $\infty$, while $f_{2 \mathrm{z}}$ oscillates near the maximum and minimum values, in contrast to the special case of $x<1$ as shown in the Fig. 3(d).

\section{BOGOLIUBOV SPECTRA AND GOLDSTONE MODES}

This section is denoted to the discussion of Bogoliubov excitations of the ground state phases we derived above. We follow the number-conserving approach initially developed by Gardiner et. al. [45], where $\hat{a}_{\mathbf{0}, m}$ $\left(\hat{b}_{\mathbf{0}, m}\right)$ is replaced by $\left(N_{1}-\sum_{\mathbf{k} \neq 0, m} \hat{a}_{\mathbf{k}, m}^{\dagger} \hat{a}_{\mathbf{k}, m}\right)^{1 / 2} \zeta_{1, m}$ $\left(\left(N_{2}-\sum_{\mathbf{k} \neq 0, m} \hat{b}_{\mathbf{k}, m}^{\dagger} \hat{b}_{\mathbf{k}, m}\right)^{1 / 2} \zeta_{2, m}\right)$ [40, 46, 47], and keeping only quadratic terms of the operators to derive the effective Hamiltonian by using the same notations as Refs. [40, 47], 


$$
\begin{aligned}
\hat{H}^{\mathrm{eff}} & =\hat{H}_{1}^{\mathrm{eff}}+\hat{H}_{2}^{\mathrm{eff}}+\hat{H}_{12}^{\mathrm{eff}}, \\
\hat{H}_{1}^{\mathrm{eff}} & =\sum_{\mathbf{k} \neq 0, m}\left(\varepsilon_{1 \mathbf{k}}-p_{1} m+p_{1}\left\langle F_{1 \mathbf{z}}\right\rangle-\beta_{1} n_{1}\left\langle\mathbf{F}_{1}\right\rangle^{2}\right) \hat{a}_{\mathbf{k}, m}^{\dagger} \hat{a}_{\mathbf{k}, m}+\beta_{1} n_{1}\left\langle\mathbf{F}_{1}\right\rangle \cdot \sum_{\mathbf{k} \neq 0, m, n} \hat{a}_{\mathbf{k}, m}^{\dagger} \mathbf{F}_{1 m n} \hat{a}_{\mathbf{k}, n} \\
& +\frac{\alpha_{1} n_{1}}{2} \sum_{\mathbf{k} \neq 0}\left(2 \hat{\mathcal{D}}_{1, \mathbf{k}}^{\dagger} \hat{\mathcal{D}}_{1, \mathbf{k}}+\hat{\mathcal{D}}_{1, \mathbf{k}} \hat{\mathcal{D}}_{1,-\mathbf{k}}+\hat{\mathcal{D}}_{1, \mathbf{k}}^{\dagger} \hat{\mathcal{D}}_{1,-\mathbf{k}}^{\dagger}\right)+\frac{\beta_{1} n_{1}}{2} \sum_{\mathbf{k} \neq 0}\left(2 \hat{\mathcal{F}}_{1, \mathbf{k}}^{\dagger} \cdot \hat{\mathcal{F}}_{1, \mathbf{k}}+\hat{\mathcal{F}}_{1, \mathbf{k}} \cdot \hat{\mathcal{F}}_{1,-\mathbf{k}}+\hat{\mathcal{F}}_{1, \mathbf{k}}^{\dagger} \cdot \hat{\mathcal{F}}_{1,-\mathbf{k}}^{\dagger}\right), \\
\hat{H}_{12}^{\mathrm{eff}} & =\frac{\beta}{2} \sum_{\mathbf{k} \neq 0, m}-\left\langle\mathbf{F}_{1}\right\rangle \cdot\left\langle\mathbf{F}_{2}\right\rangle\left(n_{2} \hat{a}_{\mathbf{k}, m}^{\dagger} \hat{a}_{\mathbf{k}, m}+n_{1} \hat{b}_{\mathbf{k}, m}^{\dagger} \hat{b}_{\mathbf{k}, m}\right)+\frac{\beta n_{1}}{2}\left\langle\mathbf{F}_{1}\right\rangle \cdot \sum_{\mathbf{k} \neq 0, m, n} \hat{b}_{\mathbf{k}, m}^{\dagger} \mathbf{F}_{2 m n} \hat{b}_{\mathbf{k}, \mathbf{n}} \\
& +\frac{\beta n_{2}}{2}\left\langle\mathbf{F}_{2}\right\rangle \cdot \sum_{\mathbf{k} \neq 0, m, n} \hat{a}_{\mathbf{k}, m}^{\dagger} \mathbf{F}_{1 m n} \hat{a}_{\mathbf{k}, \mathbf{n}}+\frac{\alpha \sqrt{n_{1} n_{2}}}{2} \sum_{\mathbf{k} \neq 0}\left(\hat{\mathcal{D}}_{1, \mathbf{k}}^{\dagger} \hat{\mathcal{D}}_{2, \mathbf{k}}+\hat{\mathcal{D}}_{1, \mathbf{k}} \hat{\mathcal{D}}_{2, \mathbf{k}}^{\dagger}+\hat{\mathcal{D}}_{1, \mathbf{k}} \hat{\mathcal{D}}_{2,-\mathbf{k}}+\hat{\mathcal{D}}_{1, \mathbf{k}}^{\dagger} \hat{\mathcal{D}}_{2,-\mathbf{k}}^{\dagger}\right) \\
& +\frac{\beta \sqrt{n_{1} n_{2}}}{2} \sum_{\mathbf{k} \neq 0}\left(\hat{\mathcal{F}}_{1, \mathbf{k}}^{\dagger} \cdot \hat{\mathcal{F}}_{2, \mathbf{k}}+\hat{\mathcal{F}}_{1, \mathbf{k}} \cdot \hat{\mathcal{F}}_{2, \mathbf{k}}^{\dagger}+\hat{\mathcal{F}}_{1, \mathbf{k}} \cdot \hat{\mathcal{F}}_{2,-\mathbf{k}}+\hat{\mathcal{F}}_{1, \mathbf{k}}^{\dagger} \cdot \hat{\mathcal{F}}_{2,-\mathbf{k}}^{\dagger}\right)+\mathcal{E}_{0},
\end{aligned}
$$

where $\mathcal{E}_{0}$ is a constant term and we have defined $\hat{\mathcal{D}}_{1, \mathbf{k}}=$ $\sum_{m} \zeta_{1, m}^{*} \hat{a}_{\mathbf{k}, m}, \hat{\mathcal{F}}_{1, \mathbf{k}}=\sum_{m, n} \zeta_{1, m}^{*} \mathbf{F}_{1 m n} \hat{a}_{\mathbf{k}, n} . \quad$ As before $\hat{H}_{2}^{\text {eff }}$ takes the same form as $H_{1}^{\text {eff }}$ except for the replacing of the subscript 1 by 2 and the operator $\hat{a}$ by $\hat{b}$.

Following the notation and approach of Ref. [40], we define operators

$$
\begin{aligned}
& \hat{\mathbf{P}}_{\mathbf{k}}=\left(\hat{a}_{\mathbf{k}, 1}, \hat{a}_{\mathbf{k}, 0}, \hat{a}_{\mathbf{k},-1}, \hat{b}_{\mathbf{k}, 1}, \hat{b}_{\mathbf{k}, 0}, \hat{b}_{\mathbf{k},-1}\right)^{T}, \\
& \hat{\mathbf{P}}_{\mathbf{k}}^{*}=\left(\hat{a}_{\mathbf{k}, 1}^{\dagger}, \hat{a}_{\mathbf{k}, 0}^{\dagger}, \hat{a}_{\mathbf{k},-1}^{\dagger}, \hat{b}_{\mathbf{k}, 1}^{\dagger}, \hat{b}_{\mathbf{k}, 0}^{\dagger}, \hat{b}_{\mathbf{k},-1}^{\dagger}\right)^{T},
\end{aligned}
$$

which satisfy the Heisenberg equation

$$
i \hbar \frac{d}{d t} \hat{\mathbf{P}}_{\mathbf{k}}=\mathcal{M}(k) \hat{\mathbf{P}}_{\mathbf{k}}+\mathcal{N}(k) \hat{\mathbf{P}}_{-\mathbf{k}}^{*},
$$

to calculate low-lying Bogoliubov modes. $\mathcal{M}(k)$ and $\mathcal{N}(k)$ are chosen as real with suitable real valued $\zeta_{1}$ and $\zeta_{2}$ and symmetric $6 \times 6$ matrix. The operators for Bogoliubov quasi-particles are defined accordingly

$$
\begin{aligned}
\hat{\mathbf{Q}}_{\mathbf{k}} & =\left(\hat{\mathcal{Q}}_{\mathbf{k}, 1}, \hat{\mathcal{Q}}_{\mathbf{k}, 2}, \hat{\mathcal{Q}}_{\mathbf{k}, 3}, \hat{\mathcal{Q}}_{\mathbf{k}, 4}, \hat{\mathcal{Q}}_{\mathbf{k}, 5}, \hat{\mathcal{Q}}_{\mathbf{k}, 6}\right)^{T} \\
& =\mathcal{U}(k) \hat{\mathbf{P}}_{\mathbf{k}}+\mathcal{V}(k) \hat{\mathbf{P}}_{-\mathbf{k}}^{*}
\end{aligned}
$$

which enforces a diagonalized effective Hamiltonian

$$
\hat{H}^{\mathrm{eff}}=\sum_{\mathbf{k}, m} E_{m} \hat{\mathcal{Q}}_{\mathbf{k}, m}^{\dagger} \hat{\mathcal{Q}}_{\mathbf{k}, m}+E_{\mathrm{vac}},
$$

where $E_{\text {vac }}$ is the vacuum state energy for the quasiparticles.

As the quasi-particle operators $\hat{\mathcal{Q}}_{\mathbf{k}, m}$ and satisfy Bose commutation relations, we obtain

$$
i \hbar \frac{d}{d t} \hat{\mathbf{Q}}_{\mathbf{k}}=E(\mathbf{k}) \hat{\mathbf{Q}}_{\mathbf{k}},
$$

where $E(\mathbf{k})$ is a diagonal $6 \times 6$ matrix: $\operatorname{diag}(E)=$ $\left(E_{1}, E_{2}, E_{3}, E_{4}, E_{5}, E_{6}\right)^{T}$. The eigenvalues of the quasiparticles are found from solving the following eigenequation [48]

$$
(\mathcal{M}+\mathcal{N})(\mathcal{M}-\mathcal{N})(\mathcal{U}+\mathcal{V})^{T}=(\mathcal{U}+\mathcal{V})^{T} E^{2} .
$$

We choose to reexamine the ground states of the Hamiltonian of Eq. (4) before discussing the Bogoliubov excitations and the Goldstone modes. As shown in the appendix $\mathrm{A}$, there exist two types of mean field phases in the ground state, one preserves axisymmetry, and the other breaks axisymmetry. The axisymmetry preserved phases include $\mathrm{FF}, \mathrm{AA}, \mathrm{P}_{+} \mathrm{N}, \mathrm{P}_{-} \mathrm{N}, \mathrm{NP}_{+}$, and NN phases, etc. All ground state phases can be reclassified into four classes according to the numbers of nonzero component in their order parameters: the $\mathrm{FF}$ and AA phases falls into the two-component class, the $\mathrm{P}_{+} \mathrm{N}, \mathrm{P}_{-} \mathrm{N}$, and $\mathrm{NP}_{+}$phases belongs to the threecomponent class, while the four-component class includes the NN phase and the six component class includes the BA phase.

According to the Goldstone theorem: a gapless mode emerges whenever a continuous symmetry is spontaneously broken. For the two-component class, two U(1) symmetries for the two atomic species are broken [49]. One and two more spin rotation symmetries are broken in both the three-component and four-component classes. For the BA phase, two U(1) symmetries and one $\mathrm{SO}(2)$ symmetry are broken [50]. To verify the above arguments, we solve the eigen-equations of Eq. (14) employing both analytic or numerical techniques. In the ground state of the Hamiltonian of Eq. (4), unequal densities of two species can modify the spinexchange interaction and linear Zeeman shifts. Without loss of generality, in the following numerical calculation, we will assume $N_{1}=N_{2}$ and $n_{1}=n_{2}=n$. Because spin-independent interactions do not change the general properties of the Bogoliubov excitations, we will set intra- and inter-species spin-independent interaction as $\alpha_{1} n_{1}=\alpha_{2} n_{2}=\alpha \sqrt{n_{1} n_{2}}=\alpha n=20\left|\beta_{1}^{\prime}\right|$ and we further assume $M_{2}=3 M_{1}$. 


\section{A. two-component class}

As summarized above, the two-component class contains two phases: the FF phase and AA phase. We first consider the FF phase by assuming $\zeta_{1}=\zeta_{2}=(1,0,0)^{T}$ and $p>0$. After detailed calculation, we find

$$
\begin{aligned}
& E_{1}^{2}=\frac{1}{2}\left(\epsilon_{1 \mathbf{k}}^{2}+\epsilon_{2 \mathbf{k}}^{2}-\sqrt{\left(\epsilon_{1 \mathbf{k}}^{2}-\epsilon_{2 \mathbf{k}}^{2}\right)^{2}+4(\alpha n+\beta n)^{2} \varepsilon_{1 \mathbf{k}} \varepsilon_{2 \mathbf{k}}}\right), \\
& E_{2}^{2}=\frac{1}{2}\left(\epsilon_{1 \mathbf{k}}^{2}+\epsilon_{2 \mathbf{k}}^{2}+\sqrt{\left(\epsilon_{1 \mathbf{k}}^{2}-\epsilon_{2 \mathbf{k}}^{2}\right)^{2}+4(\alpha n+\beta n)^{2} \varepsilon_{1 \mathbf{k}} \varepsilon_{2 \mathbf{k}}}\right), \\
& E_{3}^{2}=\frac{1}{4}\left(\varepsilon_{1 \mathbf{k}}+\varepsilon_{2 \mathbf{k}}-\beta n+p_{1}+p_{2}-\sqrt{\left(\varepsilon_{1 \mathbf{k}}-\varepsilon_{2 \mathbf{k}}+p_{1}-p_{2}\right)^{2}+(\beta n)^{2}}\right)^{2}, \\
& E_{4}^{2}=\frac{1}{4}\left(\varepsilon_{1 \mathbf{k}}+\varepsilon_{2 \mathbf{k}}-\beta n+p_{1}+p_{2}+\sqrt{\left(\varepsilon_{1 \mathbf{k}}-\varepsilon_{2 \mathbf{k}}+p_{1}-p_{2}\right)^{2}+(\beta n)^{2}}\right)^{2}, \\
& E_{5}^{2}=\left(\varepsilon_{1 \mathbf{k}}-2 \beta_{1} n-\beta n+2 p_{1}\right)^{2}, \\
& E_{6}^{2}=\left(\varepsilon_{2 \mathbf{k}}-2 \beta_{2} n-\beta n+2 p_{2}\right)^{2},
\end{aligned}
$$

where $\epsilon_{1 \mathbf{k}}^{2}=\varepsilon_{1 \mathbf{k}}^{2}+2\left(\alpha_{1} n+\beta_{1} n\right) \varepsilon_{1 \mathbf{k}}$ and $\epsilon_{2 \mathbf{k}}^{2}=\varepsilon_{2 \mathbf{k}}^{2}+$ $2\left(\alpha_{2} n+\beta_{2} n\right) \varepsilon_{2 \mathbf{k}}$. From Eq. (15), we confirm that there are two gapless Goldstone modes with eigenvalues $E_{1}$ and $E_{2}$, associated with the two coupled $\mathrm{U}(1)$ symmetry breaking modes between the two $M_{F}=1$ components of two species respectively. The two coupled modes between the two $M_{F}=0$ components are gapped, and their excitation spectra are denoted by $E_{3}$ and $E_{4}$. The remaining two excitation spectra are the two modes coupling be- tween two $M_{F}=-1$ components, which are gapped as well. In the boundary between the FF phase and the BA phase, where $|p|=\beta n$, we find the excitation spectra of $E_{3}$ reduce to a free particle form at small values of momentum $k$.

For the AA phase, without loss of generality, we can assume the order parameters of the forms $\zeta_{1}=(1,0,0)^{T}$ and $\zeta_{2}=(0,0,1)^{T}$. The excitation spectra can be analytically retrieved in this case as

$$
\begin{aligned}
& E_{1}^{2}=\frac{1}{2}\left(\epsilon_{1 \mathbf{k}}^{2}+\epsilon_{2 \mathbf{k}}^{2}-\sqrt{\left(\epsilon_{1 \mathbf{k}}^{2}-\epsilon_{2 \mathbf{k}}^{2}\right)^{2}+4(\alpha n-\beta n)^{2} \varepsilon_{1 \mathbf{k}} \varepsilon_{2 \mathbf{k}}}\right) \\
& E_{2}^{2}=\frac{1}{2}\left(\epsilon_{1 \mathbf{k}}^{2}+\epsilon_{2 \mathbf{k}}^{2}+\sqrt{\left(\epsilon_{1 \mathbf{k}}^{2}-\epsilon_{2 \mathbf{k}}^{2}\right)^{2}+4(\alpha n-\beta n)^{2} \varepsilon_{1 \mathbf{k}} \varepsilon_{2 \mathbf{k}}}\right) \\
& E_{3}^{2}=\frac{1}{4}\left(\varepsilon_{1 \mathbf{k}}-\varepsilon_{2 \mathbf{k}}+p_{1}+p_{2}-\sqrt{\left(\varepsilon_{1 \mathbf{k}}+\varepsilon_{2 \mathbf{k}}+\beta n+p_{1}-p_{2}\right)^{2}-(\beta n)^{2}}\right)^{2}, \\
& E_{4}^{2}=\frac{1}{4}\left(\varepsilon_{1 \mathbf{k}}-\varepsilon_{2 \mathbf{k}}+p_{1}+p_{2}+\sqrt{\left(\varepsilon_{1 \mathbf{k}}+\varepsilon_{2 \mathbf{k}}+\beta n+p_{1}-p_{2}\right)^{2}-(\beta n)^{2}}\right)^{2}, \\
& E_{5}^{2}=\left(\varepsilon_{1 \mathbf{k}}-2 \beta_{1} n+\beta n+2 p_{1}\right)^{2}, \\
& E_{6}^{2}=\left(\varepsilon_{2 \mathbf{k}}-2 \beta_{2} n+\beta n-2 p_{2}\right)^{2} .
\end{aligned}
$$

Again there are two gapless Goldstone modes, associated with the breaking of the two coupled U(1) symmetries between the components of $\zeta_{1,1}$ and $\zeta_{2,-1}$ denoted by $E_{1}$ and $E_{2}$. The two coupled gapped modes of the $M_{F}=0$ spin components for the two species are denoted as $E_{3}$ and $E_{4}$. The remaining two gapped modes come from the coupling between the components of $\zeta_{1,-1}$ and $\zeta_{2,1}$. All results are consistent with our previous discussions.

\section{B. three-component class}

For the three-component class, the spin vector of one species is fully polarized while the other one is partially polarized, resulting in the breaking of two U(1) symmetries and one $\mathrm{SO}(2)$ spin rotation symmetry. For all phases: $\mathrm{P}_{+} \mathrm{N}, \mathrm{P}_{-} \mathrm{N}$, and $\mathrm{NP}_{+}$, their Bogoliubov spectra contain three gapless Goldstone modes. We verify the above results numerically by diagonalizing Eq. (14). The case of the $\mathrm{P}_{+} \mathrm{N}$ is shown for illustrative purposes 


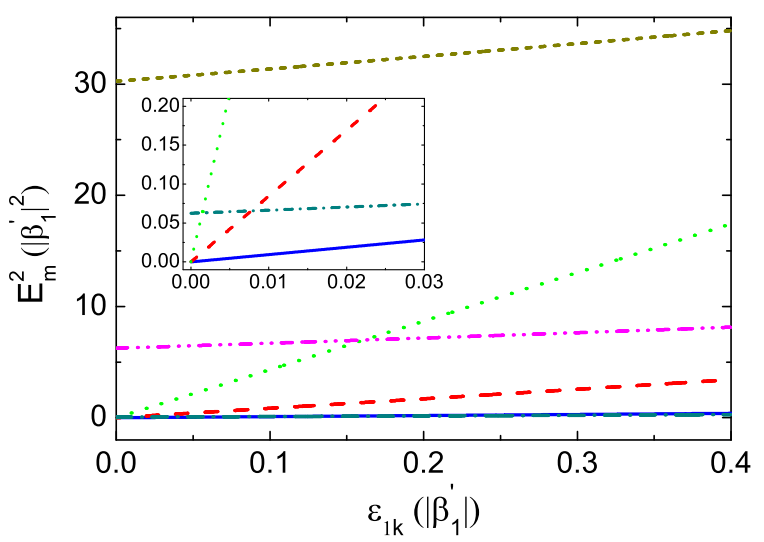

FIG. 5: (Color online). The Bogoliubov excitation spectra for the $\mathrm{P}_{+} \mathrm{N}$ phase. The intra- and inter-species spin-dependent interaction parameters used are $\beta_{1}^{\prime}<0, \beta_{2}^{\prime}=2\left|\beta_{1}^{\prime}\right|$, and $\beta^{\prime}=$ $3\left|\beta_{1}^{\prime}\right|$. The B-field parameters are $p_{1}=p_{2}=2.5\left|\beta_{1}^{\prime}\right|$. The inset shows the small momentum $k$ region.

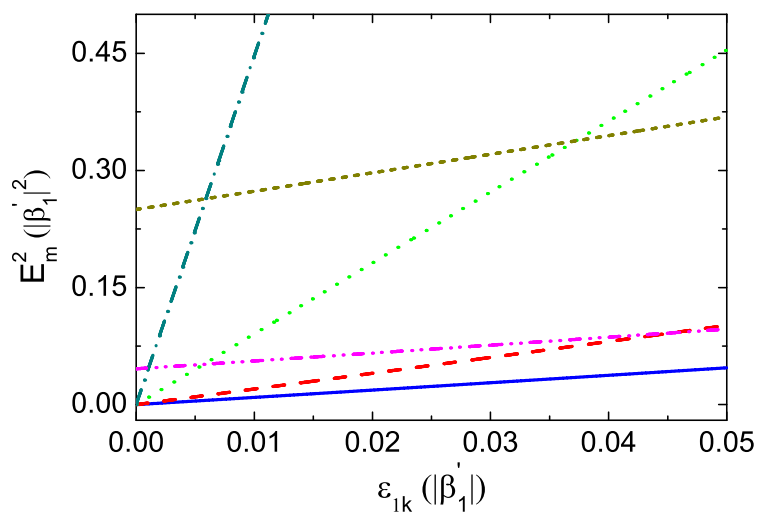

FIG. 6: (Color online). The same as in Fig. 5 but for the $\mathrm{NN}$ phase. The parameters adopted are $\beta_{1}^{\prime}>0, \beta_{2}^{\prime}=2\left|\beta_{1}^{\prime}\right|$, $\beta^{\prime}=\left|\beta_{1}^{\prime}\right|$, and $p_{1}=p_{2}=\left|\beta_{1}^{\prime}\right| / 2$.

in Fig. 5, where we assume spin-dependent parameters $\beta_{1}^{\prime}<0, \beta_{2}^{\prime}=2\left|\beta_{1}^{\prime}\right|, \beta^{\prime}=3\left|\beta_{1}^{\prime}\right|$, and linear Zeeman shifts $p_{1}=p_{2}=2.5\left|\beta_{1}^{\prime}\right|$. The order parameters take the forms of Eq. (6), where now we have $f_{1 \mathrm{z}}=1$ and we choose $\chi_{1}=\chi_{2}=\varphi_{1}=\varphi_{2}=0$.

\section{C. four-component class}

There exists only a single one phase: the NN phase, for the four-component class. Its order parameter takes the form of Eq. (6). For simplicity, we assume $\chi_{1}=$ $\chi_{2}=\varphi_{1}=\varphi_{2}=0$. In Fig. [6 we illustrates its collective excitation spectra, where the intra- and inter-species spin-dependent interactions are parameterized by $\beta^{\prime}>0$, $\beta_{2}^{\prime}=2\left|\beta_{1}^{\prime}\right|$, and $\beta^{\prime}=\left|\beta_{1}^{\prime}\right|$. The B-field parameters are chosen as $p_{1}=p_{2}=\left|\beta_{1}^{\prime}\right| / 2$. Indeed one can infer that there are four gapless Goldstone modes in Fig. 6, consistent with the previous discussions.

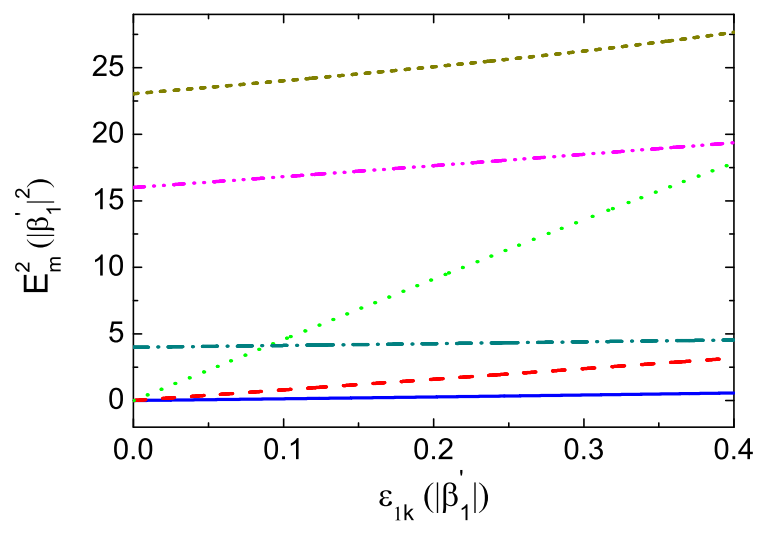

FIG. 7: (Color online). The same as in Fig. 5 but for the $\mathrm{BA}$ phase. The parameters adopted are $\beta_{1}^{\prime}>0, \beta_{2}^{\prime}=2\left|\beta_{1}^{\prime}\right|$, $\beta^{\prime}=6\left|\beta_{1}^{\prime}\right|$, and $p_{1}=p_{2}=4 \beta^{\prime} / 5$.

\section{D. six-component class}

Finally for the six component class, the axisymmetry is broken and its order parameter taking the form of Eq. (7). Due to breaking of two $\mathrm{U}(1)$ and one $\mathrm{SO}(2)$ symmetries, the Bogoliubov excitation spectra contain three gapless Goldstone modes, as confirmed by numerically solving Eq. (14). $\chi_{1}=\chi_{2}=\varphi_{1}=0$ and $\varphi_{2}=\pi$ are used for numerical calculations, together with the intra- and interspecies spin-dependent parameters $\beta_{1}^{\prime}>0, \beta_{2}^{\prime}=2\left|\beta_{1}^{\prime}\right|$, and $\beta^{\prime}=6\left|\beta_{1}^{\prime}\right|$. The linear Zeeman shifts are assumed to satisfy $p_{1}=p_{2}=4 \beta^{\prime} / 5$. In Fig. 7 we present all Bogoliubov excitation spectra, from which one can easily verify the three gapless Goldstone modes.

\section{CONCLUSIONS}

In conclusion, we construct the ground state phase diagram for a binary mixture of two spin-1 condensates under an external weak B-field. Our results show that the phase diagram can be classified into two categories: the axisymmetry preserved and broken phases. The first one is shown to contain the FF, AA, $\mathrm{P}_{+} \mathrm{N}, \mathrm{P}_{-} \mathrm{N}, \mathrm{NP}_{+}$, and NN phases, while the second one contains only single phase: the BA phase, which appears only when the inter-species anti-ferromagnetic spin-exchange interaction is sufficiently large when compared to the intraspecies spin-exchange and linear Zeeman shifts. Furthermore, according to the number of the gapless Goldstone modes, the ground state phases can be classified into four classes: two-, three-, four-, and six-component class, respectively accompanied by two, three, four, and three gapless Goldstone modes. We calculate all the Bogoliubov excitation spectra with both analytical or numerical techniques, and confirm our arguments given above.

Since the interspecies s-wave interaction parameters between two spin- 1 atoms, such as between ${ }^{87} \mathrm{Rb}$ and ${ }^{23} \mathrm{Na}$ atoms, are not precisely known, we cannot con- 
clude which of the above discussed phases can be realized experimentally within presently available experimental technologies. Given the promising innovation of tuning interspecies interaction parameters with optical Feshbach resonances and other tools, it seems that phases we study can stimulate sufficient experimental interests to confirm their existence.

\section{ACKNOWLEDGMENTS}

This work is supported by the NSF of China under Grant Nos. 11004116, 10640420151 and 10974112 and by NKBRSF of China under Grant Nos. 2006CB921206, 2006AA06Z104, 2006CB605105 and 2006CB921404.

\section{Appendix A: constructing the ground state phases}

In this appendix, we give the detail discussion on how to clarify all possible ground state phases for a binary mixture of spin-1 condensates under an external weak B-field.

First, we assume that spontaneous axisymmetry breaking doest not occur in either species, which results in $f_{1 \pm}=f_{2 \pm}=0$. For species one, we then have

$$
\sqrt{2}\left(\zeta_{1}^{(1) *} \zeta_{0}^{(1)}+\zeta_{0}^{(1) *} \zeta_{-1}^{(1)}\right)=0
$$

for the ground state. Without loss of generality, an unitary transformation can be affected to set $\zeta_{0}^{(1)}$ as a real number. As a result, there left only two solutions for Eq. (A1): $\zeta_{0}^{(1)}=0$ or $\zeta_{1}^{(1) *}+\zeta_{-1}^{(1)}=0$. For the latter case, we infer that $\left|\zeta_{1}^{(1)}\right|^{2}=\left|\zeta_{-1}^{(1)}\right|^{2}$ and $f_{1 \mathrm{z}}=0$, which is a state degenerate with $\zeta_{1}=\left(e^{i \varphi_{1}}, 0, e^{i \varphi_{-1}}\right)^{T} / \sqrt{2}$, with arbitrary angles $\varphi_{1}$ and $\varphi_{-1}$. Therefore the ground states is limited to the subspace with $\zeta_{0}^{(1)}=0$. For the second species, an analogous reduction gives a similar subspace with $\zeta_{0}^{(2)}=0$. Summarizing, without spontaneous axisymmetry breaking, the ground states of the energy functional for a binary mixture of two spin-1 condensates, Eq. (5), can be chosen as $\zeta_{1}=\left(\zeta_{1}^{(1)}, 0, \zeta_{-1}^{(1)}\right)^{T}$ and $\zeta_{2}=\left(\zeta_{1}^{(2)}, 0, \zeta_{-1}^{(2)}\right)^{T}$.
Next, we consider the structure of all possible spontaneous axisymmetry breaking ground states. For a single spin-1 condensate, the linear Zeeman shift does not induce BA phase. If a spontaneous BA phase arises in a mixture, both species must break axisymmetry. We therefore search for the ground states of the BA phase in the subspace of $\mathbf{f}_{1}^{2}=a^{2}$ and $\mathbf{f}_{2}^{2}=b^{2}$, with $a^{2} \leq 1$ and $b^{2} \leq 1$. Since $p_{1} p_{2} \geq 0$, the BA phase appears for anti-ferromagnetic interspecies spin-exchange interaction with $\beta^{\prime}>0$. In the ground states, the transverse magnetization of the two species must align along opposite directions.

The order parameter for the BA phase can be found from the following equations

$$
\begin{aligned}
& \frac{d \mathcal{E}_{s}}{d f_{1 \mathrm{z}}}=-p+\frac{1}{2} \beta^{\prime}\left(f_{2 \mathrm{z}}+\frac{\sqrt{b^{2}-f_{2 \mathrm{z}}^{2}}}{\sqrt{a^{2}-f_{1 \mathrm{z}}^{2}}} f_{1 \mathrm{z}}\right)=0, \\
& \frac{d \mathcal{E}_{s}}{d f_{2 \mathrm{z}}}=-x p+\frac{1}{2} \beta^{\prime}\left(f_{1 \mathrm{z}}+\frac{\sqrt{a^{2}-f_{1 \mathrm{z}}^{2}}}{\sqrt{b^{2}-f_{2 \mathrm{z}}^{2}}} f_{2 \mathrm{z}}\right)=0 .
\end{aligned}
$$

The solution of the above equations for the BA phase is given by $f_{1 \mathrm{z}}=x p / \beta^{\prime}+a^{2} \beta^{\prime} / 4 x p-x b^{2} \beta^{\prime} / 4 p$ and $f_{2 \mathrm{z}}=$ $p / \beta^{\prime}+b^{2} \beta^{\prime} / 4 p-a^{2} \beta^{\prime} / 4 x^{2} p$. We can calculate the first derivatives of the spin-dependent energy function $\mathcal{E}_{s}$ with respect to $a^{2}$ and $b^{2}$ at the BA phase, and the results are

$$
\begin{aligned}
& \frac{d \mathcal{E}_{s}}{d a^{2}}=\frac{1}{2} \beta_{1}^{\prime}-\frac{1}{2} \beta^{\prime} \frac{\sqrt{b^{2}-f_{2 \mathrm{z}}^{2}}}{2 \sqrt{a^{2}-f_{1 \mathrm{z}}^{2}}}=\frac{1}{4}\left(2 \beta_{1}^{\prime}-\beta^{\prime} / x\right), \\
& \frac{d \mathcal{E}_{s}}{d b^{2}}=\frac{1}{2} \beta_{2}^{\prime}-\frac{1}{2} \beta^{\prime} \frac{\sqrt{a^{2}-f_{1 \mathrm{z}}^{2}}}{2 \sqrt{b^{2}-f_{2 \mathrm{z}}^{2}}}=\frac{1}{4}\left(2 \beta_{2}^{\prime}-\beta^{\prime} x\right) .
\end{aligned}
$$

We therefore conclude that the BA phase is possiblely exist only when $\beta^{\prime} \geq \max \left(0,2 \beta_{1}^{\prime} x, 2 \beta_{2}^{\prime} / x\right)$. Because the first derivatives are both independent of $a^{2}$ and $b^{2}$, the ground states of the BA phase will have $a^{2}=b^{2}=1$.

The actual ground state is found through a comparison of the ground state energies for two types of phases: with/without broken-axisymmetry. In addition, the results found here are subsequently affirmed with the numerical ones from the use of simulated annealing method 36] to minimize the spin-dependent energy functional.
[1] Masahito Ueda and Yuki Kawaguchi, arXiv: 1001.2072.

[2] D. M. Stamper-Kurn, M. R. Andrews, A. P. Chikkatur, S. Inouye, H.-J. Miesner, J. Stenger, and W. Ketterle, Phys. Rev. Lett. 80, 2027 (1998).

[3] J. Stenger et al., Nature (London) 396, 345 (1998).

[4] M. Barrett, J. Sauer, and M. S. Chapman, Phys. Rev. Lett. 87, 010404 (2001).

[5] H. Schmaljohann, M. Erhard, J. Kronjäger, M. Kottke, S. van Staa, L. Cacciapuoti, J. J. Arlt, K. Bongs, and K. Sengstock, Phys. Rev. Let. 92, 040402 (2004).
[6] Axel Griesmaier, Jörg Werner, Sven Hensler, Jürgen Stuhler, and Tilman Pfau, Phys. Rev. Lett. 94, 160401 (2005).

[7] Tin-Lun Ho, Phys. Rev. Lett. 81, 742 (1998).

[8] T. Ohmi and K. Machida, J. Phys. Soc. Jpn. 67, 1822 (1998).

[9] C. K. Law, H. Pu, and N. P. Bigelow, Phys. Rev. Lett. 81, 5257 (1998).

[10] J. W. Reijnders, F. J. M. van Lankvelt, K. Schoutens, and N. Read, Phys. Rev. A 69, 023612 (2004). 
[11] Erich J. Mueller, Phys. Rev. A 69, 033606 (2004).

[12] Masato Koashi and Masahito Ueda, Phys. Rev. Lett. 84, 1066 (2000).

[13] Tin-Lun Ho and Sung Kit Yip, Phys.Rev.Lett. 84, 4031 (2000).

[14] Fei Zhou, Phys. Rev. Lett. 87, 080401 (2001).

[15] Wenxian Zhang, D. L. Zhou, M.-S. Chang, M. S. Chapman, and L. You, Phys. Rev. Lett. 95, 180403 (2005)

[16] L. E. Sadler, J. M. Higbie, S. R. Leslie, M. Vengalattore, D. M. Stamper-Kurn, Nature 443, 312 (2006).

[17] Wenxian Zhang, D. L. Zhou, M.-S. Chang, M. S. Chapman, and L. You, Phys. Rev. A 72, 013602 (2005).

[18] Lee Chang, Q. Zhai, Rong Lu, and L. You, Phys. Rev. Lett. 99, 080402 (2007).

[19] Ming-Shien Chang, Qishu Qin, Wenxian Zhang, Li You, Michael S. Chapman, Nature Physics 1, 111 (2005).

[20] Y. Liu, S. Jung, S. E. Maxwell, L. D. Turner, E. Tiesinga, and P. D. Lett, Phys. Rev. Lett. 102, 125301 (2009).

[21] C. V. Ciobanu, S.-K.Yip, and Tin-Lun Ho, Phys. Rev. A 61, 033607 (2000).

[22] M. Ueda and M. Koashi, Phys. Rev. A 65, 063602 (2002).

[23] H. Mäkelä, Y. Zhang, and K.-A. Suominen, J. Phys. A: Math. Gen. 36, 8555 (2003).

[24] Gordon W. Semenoff and Fei Zhou, Phys. Rev. Lett. 98, 100401 (2007).

[25] Michikazu Kobayashi, Yuki Kawaguchi, Muneto Nitta, and Masahito Ueda, Phys. Rev. Lett. 103, 115301 (2009).

[26] Artur Widera, Fabrice Gerbier, Simon Fölling, Tatjana Gericke, Olaf Mandel and Immanuel Bloch, New J. Phys. 8, 152 (2006).

[27] J. Kronjäger, C. Becker, P. Navez, K. Bongs, and K. Sengstock, Phys. Rev. Lett. 97, 110404 (2006).

[28] Roberto B. Diener and Tin-Lun Ho, Phys. Rev. Lett. 96, 190405 (2006).

[29] L. Santos and T. Pfau, Phys. Rev. Lett. 96, 190404 (2006).

[30] D. S. Hall, M. R. Matthews, J. R. Ensher, C. E. Wieman, and E. A. Cornell, Phys. Rev. Lett. 81, 1539 (1998).

[31] G. Thalhammer, G. Barontini, L. De Sarlo, J. Catani, F. Minardi, and M. Inguscio, Phys. Rev. Lett. 100, 210402 (2008).

[32] S. B. Papp, J. M. Pino, and C. E. Wieman, Phys. Rev. Lett. 101, 040402 (2008).
[33] A. B. Kuklov and B. V. Svistunov, Phys. Rev. Lett. 89, 170403 (2002).

[34] S. Ashhab and A. J. Leggett, Phys. Rev. A 68, 063612 (2003).

[35] Yu Shi and Qian Niu, Phys. Rev. Lett. 96, 140401 (2006).

[36] Z. F. Xu, Yunbo Zhang, and L. You, Phys. Rev. A 79, 023613 (2009).

[37] Z. F. Xu, Jie Zhang, Yunbo Zhang, and L. You, Phys. Rev. A 81, 033603 (2010).

[38] Jie Zhang, Z. F. Xu, L. You, and Yunbo Zhang, Phys. Rev. A 82, 013625 (2010).

[39] Yu Shi, Phys. Rev. A 82, 023603 (2010).

[40] Keiji Murata, Hiroki Saito, and Masahito Ueda, Phys. Rev. A 75, 013607 (2007).

[41] S. B. Weiss, M. Bhattacharya, and N. P. Bigelow, Phys. Rev. A 68, 042708 (2003).

[42] A. Pashov, O. Docenko, M. Tamanis, R. Ferber, H. Knöckel, and E. Tiemann, Phys. Rev. A 72, 062505 (2005).

[43] H. T. C. Stoof, J. M. V. A. Koelman, and B. J. Verhaar, Phys. Rev. B 38, 4688 (1988).

[44] Ma Luo, Zhibing Li, and Chengguang Bao, Phys. Rev. A 75, 043609 (2007).

[45] C. W. Gardiner, Phys. Rev. A 56, 1414 (1997); S. A. Gardiner and S. A. Morgan, Phys. Rev. A 75, 043621 (2007).

[46] Y. Castin and R. Dum, Phys. Rev. A 57, 3008 (1998); Y. Castin, in 'Coherent atomic matter waves', Lecture Notes of Les Houches Summer School, p.1-136, edited by R. Kaiser, C. Westbrook, and F. David, EDP Sciences and Springer-Verlag (2001)

[47] Masahito Ueda, Phys. Rev. A 63, 013601 (2000).

[48] L. You, W. Hoston, M. Lewenstein, and M. Marinescu, proceedings of the International Conference: Quantum Optics IV, (Jaszowiec, Poland, 1997), Editors, M. Kolwas and J. Mostowski, Acta Physica Polonica A 93, 211 (1998).

[49] M. Lewenstein and L. You, Phys. Rev. Lett. 77, 3489 (1996).

[50] S. Yi, Ö. E. Müstecaplığlu, and L. You, Phys. Rev. Lett. 90, 140404 (2003). 